\title{
Cauda Equina Syndrome from Subdural Hematoma after Caudal Epidural Injection
}

\author{
Jung Jae Oh, Jong-Hyeok Park, Jong Tae Kim \\ Department of Neurosurgery, Incheon St. Mary's Hospital, The Catholic University of Korea, Incheon, Republic of Korea
}

Corresponding author:

Jong-Hyeok Park

Department of Neurosurgery,

Incheon St. Mary's Hospital, The

Catholic University of Korea, 56

Dongsu-ro, Bupyeong-gu, Incheon

21431, Republic of Korea

Tel: +82-32-280-5973

Fax: +82-32-280-5991

E-mail: maimidiary@naver.com

Received: April 15, 2021

Revised: May 29, 2021

Accepted: June 2, 2021

\begin{abstract}
Caudal epidural injection (CEI) is commonly used to manage patients with low back or leg pain, but cauda equine syndrome (CES) rarely results from epidural and/or subdural hematoma occurred after the procedure. A 71-year-old man presented at our outpatient department with voiding difficulty and radiating pain in both legs 8 days ago after CE. The patient also complained pain and numbness of saddle area, CES. He had taken clopidogrel for 8 years after cardiac stent insertion. Lumbar magnetic resonance imaging (MRI) revealed subdural hematoma at the L5-S1-S2 level. The patient underwent urgent surgical treatment for hematoma evacuation. We finally recognized that subdural hematoma in lumbar MRI was found to subdural-extra arachnoid hematoma as considering intraoperative findings. To the best of our knowledge, few cases of this complication have been reported after CEI. Subdural hematoma often is concomitant with subarachnoid hematoma. Subdural and subarachnoid hematoma is associated with poor clinical outcomes due to severe edema of nerve rootlets. However, no evidence of edema and swelling of nerve rootlets was observed during surgery in our case. As a result, the patient achieved full recovery more than we expected unlike the case of subdural and subarachnoid hematoma.
\end{abstract}

Key Words: Subdural hematoma; Caudal epidural injection; Cauda equina syndrome; Voiding difficulty

\section{INTRODUCTION}

Caudal epidural injection (CEI) is commonly used to manage patients with low back and leg pain and is simple and safe, and has broad indications"). Nevertheless, complications vanying from minor to major, such as hypotension, bradycardia, transient headache, epidural or subdural hematoma, cauda equine syndrome (CES), or paraplegia, can develop affer the procedure ${ }^{3}$. Some authors have reported subdural hematoma occurred after the procedure, ${ }^{5,8,9}$. However, to the best of our knowledge, few case reports have been released on subdural-extra arachnoid hematoma after CEI. Here, we describe our experience of the surgical treatment of subdural-extra arachnoid hematoma occurring after CEI.

\section{CASE REPORT}

A 71-year-old man complaining of voiding difficulties and radiating pain in both legs visited our outpatient deparment. He had undergone cardiac stent insertion 8 years previously and had been taken clopidogrel afterward. He had received CEI for low back and right lateral calf radiating pain at another hospital nine days ago. However, low back and right leg pain was aggravated, left leg pain was newly developing after CEI. The next day the patient recognized voiding and defecation difficulties, and felt pain and numbness of saddle area such as CES. The patient waited for days expecting to get bet- ter as taking the medicine, but it did not improve. When he revisited the hospital, the doctor recommended our hospital for further evaluation. In neurologic examination, weakness of both legs was absent, but pain and numbness of both posterior thigh and saddle area. Deep tendon reflex was within normal limit. Anal tone was decreased slightly in rectal exam. Lumbar magnetic resonance imaging (MRI) showed subdural hematoma at the L5-S1-S2 level, and computed tomography depicted high density at the same level (Fig. 1). A red pin point was found at coccygeal area as predicting the insertion point of caudal block. The patient underwent emergent surgical treatment for hematoma evacuation at L5-S1-S2 level. A dark brown thecal sac was found after L5 left hemilaminectomy and S1 total laminectomy, and S2 bilateral partial laminectomy (Fig. 2A). Dural incision was performed meticulously, and subdural hematoma was removed, but the arachnoid membrane was intact and clear cerebrospinal fluid (CSF) was observed (Fig. 2B). After CSF gushed out, the dark brown hemorhage was observed at the bottom of surgical field (Fig. 2C). We found that hematoma occupied the region between the subdural and extra arachnoid spaces. The arachnoid membrane at the bottom side was dissected using a blunt hook, and subdural hematoma completely removed (Fig. 2D). Dura was meticulously sutured using the water-tight method and the suture-site was reinforced using fat tissue and fibrin glue. The surgery was completed without any complications. At postoperative day (POD) one, both leg radiating pain disappeared and the numbness of saddle area was subsided. The patient was absolute bed rest for 6 days because CSF leakage was observed at drain bag. After bed 


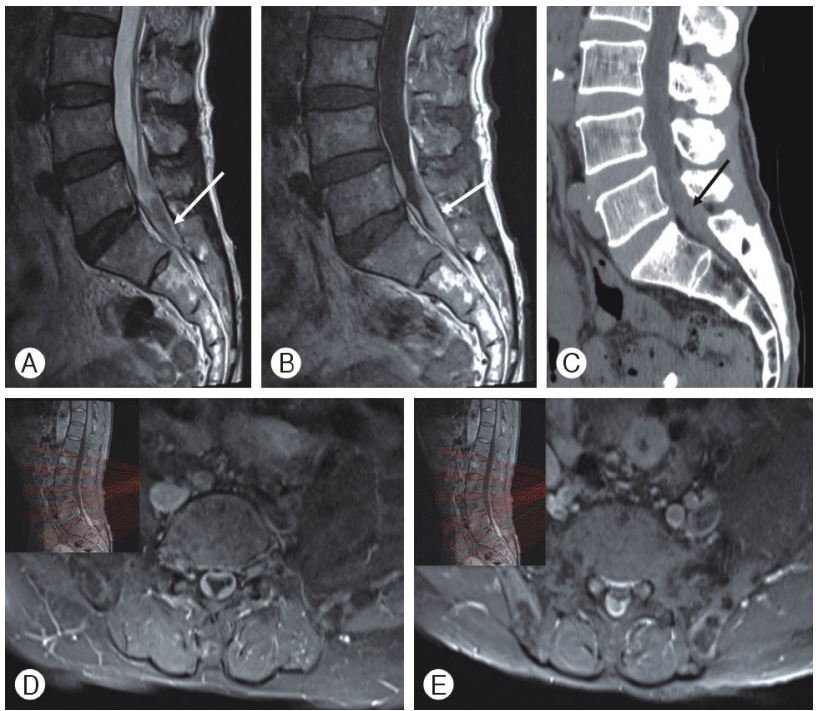

Fig. 1. (A, B) Preoperative lumbar magnetic resonance imaging (MRI) and computer tomography (CT) images. Sagittal T2 and T1-enhanced lumbar MRI images (the white arrow indicates subdural hemorrhage). (C) Sagittal CT image showing a high-density lesion in the thecal sac (black arrow). (D, E) Axial T1-enhanced lumbar MRI images showing a stellar-shaped cauda equine compressed by subdural hematoma at L5 and S1 levels.

rest, the foley catheter was removed, and the patient started to ambulate. The voiding and defecation difficulty improved incrementally at POD8, and finally voiding and defecation difficulties, and leg pain had completely resolved without any medication at POD14.

\section{DISCUSSION}

Spinal subdural hematoma is a rare complication after CEl, and sometimes it manifests as a discordant lesion from the site of injection ${ }^{5}$. Several factors such as anticoagulation, a wet-tap violating the thecal sac, high-pressure injection into sacral hiatus, or vascular anomalies, are known to contribute to the risk of subdural hematoma ${ }^{4)}$.

In our case, a dark brown thecal sac was observed in the surgical field, but CSF was transparent and the arachnoid membrane was intact. We intraoperatively found out subdural-extra arachnoid hematoma, which was not revealed on lumbar MRI (Fig. 3). We believe that a wet-tap occurred during the procedure and the needle tip violated the interspace between dura and arachnoid, and caused subdural-extra arachnoid hematoma. Moreover, the anticoagulant (clopidogrel) probably facilitated bleeding at the wet-tap site. Spinal subdural hematoma may occur in several ways, though we believe the above explanation is most plausible in our case. Therefore, we recommend the needle tip does not exceed the upper plate of the Sacral third segment to prevent wet-tap and that iodine epidurography should also be performed before CEl.

In our experience, it is difficult to recover CES that have lasted for more than one week. Nevertheless, symptoms resolved well in
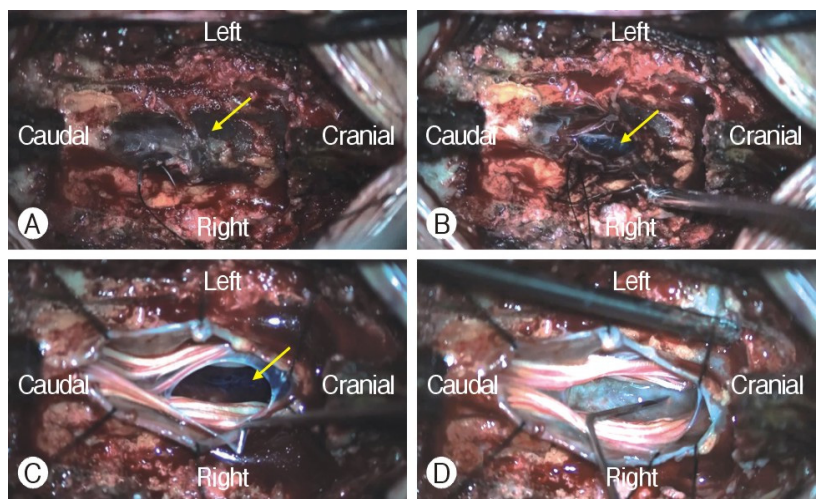

Fig. 2. (A) Intraoperative photographs. A dark brown thecal sac (yellow arrow) with an intact arachnoid membrane was observed after total laminectomy. (B) Dura is incised meticulously. Some subdural hematoma is removed. Yellow arrow designates intact arachnoid membrane and transparent cerebrospinal fluid (CSF). Subdural hematoma is observed at the bottom side through the CSF. (C) After CSF gushes out, subduralextrarachnoid hematoma (yellow arrow) was found at the bottom side. (D) After the hemorrhage at the bottom side is removed, there is not observed the edema of nerve rootlets.

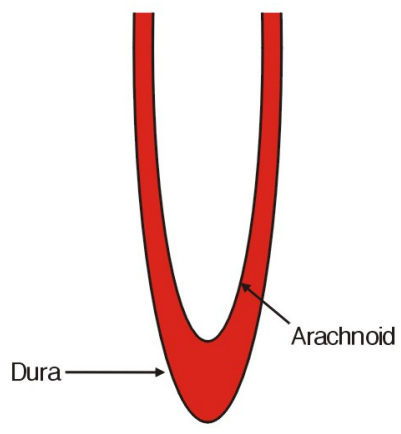

Fig. 3. A brief illustration of intraoperative finding for subdural-extrarachnoid hematoma.

our patient. In our opinion, the clinical outcomes of subdural hematoma depend on whether the hematoma occurs within or outside the arachnoid membrane. Subdural and subarachnoid hematoma usually has poor clinical outcomes resulting from severe edema and swelling of nerve rootlets. Moreover, there are the high possibility that the remnants of the hematoma cause an inflammatory response and adhesion of rootles later. However, these findings was not obser ved during surgery in our case, which probably explains the betterthan-expected clinical outcome achieved. Accordingly, clinical outcomes of spinal subdural hematoma would vary depending on the violation of arachnoid membrane.

There were several case reports that stated similar complication after CEl. Occurring CES after CEl can be caused by various pathologies. We summarized case reports in Table 1. Lee et al. ${ }^{6)}$ reported that existing herniated intervertebral disc and injected fluid could cause CES after CEI. Bilir and Gulec ${ }^{2)}$ stated that space-occupied lesion formed by injected fluid would make transient neurologic deficit. Seo et al. ${ }^{7}$ also reported that neurotoxicity came from local anesthetics could induce CES. The patient was left with a permanent neurological deficit despite of emergent surgical treatment. Chen 
Table 1. Literature review of CES after caudal epidural injection

\begin{tabular}{|c|c|c|c|c|c|}
\hline References & Year & Symptoms & Pathology & Treatment & Clinical outcomes \\
\hline Lee et al. ${ }^{6}$ & 1999 & CES + motor weakness & Herniated disc + injected fluid & Surgery & Recovery \\
\hline Bilir and Gulec ${ }^{2}$ & 2006 & CES + motor weakness & Compressive lesion by injected fluid & Conservative care & Recovery \\
\hline Seo et al. ${ }^{7}$ & 2017 & CES + motor weakness & Neurotoxicity & Surgery & Permanent deficit \\
\hline Chen et al. ${ }^{4}$ & 2020 & CES + motor weakness & Subdural hematoma & Surgery & Recovery \\
\hline Current study & 2021 & CES & Subdural-extrarachnoid hematoma & Surgery & Recovery \\
\hline
\end{tabular}

CES: cauda equina syndrome.

et al. ${ }^{4)}$ stated that subdural hematoma resulting from tearing fragile vessel by high pressure-injection could cause CES. Finally, we added our case that subdural-extra arachnoid hematoma might be formed after CEl could be one reason of CES. Furthermore, clinical outcome may especially be good in this case.

\section{CONCLUSION}

Subdural-extra arachnoid hematoma is a rare complication after CEI. Relatively good clinical outcomes could be obtained more than expected when it was not accompanied by severe edema and swelling of nerve rootlets.

\section{CONFLICTS OF INTEREST}

No potential conflict of interest relevant to this article was reported.

\section{REFERENCES}

1. Abdi S, Datta S, Lucas LF: Role of epidural steroids in the management of chronic spinal pain: A systematic review of effectiveness and complications. Pain Physician 8:127-143, 2005

2. Bilir A, Gulec S: Cauda equina syndrome after epidural steroid injection: A case report. J Manipulative Physiol Ther 29:492.e1- 492.e3, 2006

3. Botwin KP, Gruber RD, Bouchlas CG, Torres-Ramos FM, Hanna A, Rittenberg J, et al.: Complications of fluoroscopically guided caudal epidural injections. Am J Phys Med Rehabil 80:416-424, 2001

4. Chen YT, Hung WT, Tsai YC, Liu YC: Spinal subdural hematoma after ultrasound-guided caudal injection for failed back surgery syndrome. Asian J Anesthesiol 58:148-150, 2020

5. Choi JJ, Chang YJ, Jung WS, Lee KC, Kim JH, Jo YY: Discordant lumbar epidural hematoma after caudal steroid injection: A case report (CARE-compliant). Medicine (Baltimore) 96:e7127, 2017

6. Lee SH, Lee SG, Choi KH, Kim TS, Kim HS, Kim KM: Cauda equina syndrome following volumetric caudal epidural steroid injection in a herniated interverterbral disc patient: A case report. Korean J Anesthesiol 37:711-714, 1999

7. Seo YT, Kong HH, Lee GJ, Bang HJ: Persistent cauda equina syndrome after caudal epidural injection under severe spinal stenosis: A case report. J Pain Res 10:1425-1429, 2017

8. Stoll A, Sanchez M: Epidural hematoma after epidural block: implications for its use in pain management. Surg Neurol 57: 235-240, 2002

9. Vandermeulen EP, Van Aken H, Vermylen J: Anticoagulants and spinal-epidural anesthesia. Anesth Analg 79:1165-1177, 1994 